Reprod. Nutr. Déveiop., 1983, 23 (1), 65-80.

\title{
Structure glycéridique du tissu adipeux périrénal de rats soumis à un régime à base d'huile d'arachide
}

\author{
J. BÉZARD, K. A. SAWADOGO
}

Laboratoire de Physiologie animale et de la Nutrition, LA 273 C.N.R.S., U.E.R. de Nutrition,

Université de Dijon, B.P. 138, 21004 Dijon Cedex, France

Summary. The triacy/glycerol structure of perirenal adipose tissue from rats fed peanut oil.

Weanling male rats were fed a balanced diet containing 15 p. 100 peanut oil by weight. The digestibility and fecal excretion of oil fatty acids were studied for 8 days at the beginning of the diet, which was then continued up to 4 months. At the end of the experiment, perirenal adipose tissues were removed and their triacylglycerols isolated and studied. They were fractionated into 8 fractions according to unsaturation and then analyzed by gas-liquid chromatography (GLC) for fatty acids and triacylglycerols. Each fraction was submitted to pancreatic lipolysis; the mono- and diacylglycerols formed were isolated and their fatty acids analyzed by GLC. The diacylglycerols were further fractionated according to unsaturation and their fatty acid composition analyzed. The triacylglycerol structure of the adipose tissue was determined using mathematical methods previously described.

The long-chain saturated fatty acids (from stearic to lignoceric) were poorly absorbed and not incorporated into the fat tissue, whereas oleic acid, the major fatty acid of the oil (54 p. 100), was highly incorporated (57 p. 100 ) into triacylglycerols. As in the diet oil, unsaturated fatty acids were mainly esterified at the 2-position. Trioleoylglycerol was the major (21 p. 100) triacylglycerol, as in the oil $(25$ p. 100). Only 14 isomers accounted for more than 1 p. 100 and together represented more than 80 p. 100 of the total. Of the 14 isomers, the first four ( 10 p. 100 or more) accounted for more than one-half of oil triacylglycerols, nameiy $56 \mathrm{p}$. 100. The triacyiglycerol structure of studied adipose tissue was very similar to that of the diet peanut oil.

\section{Introduction.}

L'huile d'arachide est très largement consommée par la population des différentes parties du monde et ses propriétés nutritionnelles ont, pour cette raison, toujours beaucoup intéressé les chercheurs de nombreux pays (Vergroesen et Gottenbos, 1975 ; étude FAO, 1977).

Or, plusieurs études ont montré que l'huile d'arachide avait des propriétés athérogènes chez différentes espèces animales, depuis le Rat (Gresham et Howard, 1960 ; Scott et al., 1964), le lapin (Scott et al., 1964 ; Imai et al., 1966 ; 
Kritchevsky et al., 1971 et 1976) jusqu'au singe rhésus (Scott et al., 1967 ; Vesselinovitch et al., 1974). Cette particularité a été attribuée à la structure glycéridique de l'huile (Kritchevsky et al., 1973 ; Myher et al., 1977), puisque l'interestérification qui redistribue les acides gras au hasard sur les trois positions du glycérol, réduit fortement le pouvoir athérogène de l'huile (Kritchevsky et al., 1973).

On peut aussi penser à la présence, dans l'huile d'arachide, d'acides gras saturés à très longue chaîne (plus de 18 atomes de carbone) dont les propriétés athérogènes et thrombogènes sont maintenant bien établies (Scott et al., 1964 ; Hornstra, 1975 ; Brisson, 1982). Ces raisons ont conduit récemment plusieurs auteurs à s'intéresser de nouveau à la composition et à la structure glycéridique de l'huile d'arachide (Kritchevsky et al., 1973; Hokes et Worthington, 1979 ; Sanders, 1979 ; Van Pee et al., 1979 ; Manganaro et al., 1981 ; Semporé et Bézard, 1982). Tous ces travaux montrent, en particulier, un positionnement très asymétrique dans les molécules de triacylglycérols des acides gras saturés à très longue chaîne, puisque ceux-ci sont localisés uniquement en position externe et surtout en position 3, tandis que les acides insaturés sont principalement estérifiés en position 2 .

A la suite de ces observations, il nous a semblé intéressant de voir si, au niveau du tissu adipeux de Rat, la structure glycéridique de l'huile d'arachide ajoutée au régime était conservée et si les acides gras à très longue chaîne y étaient incorporés. Nous avons, pour cela, soumis des rats à un régime prolongé riche en huile d'arachide et analysé la structure des triacylglycérols d'une formation adipeuse. Cette étude nous permettait par ailleurs de comparer la structure glycéridique d'un tissu adipeux à celle de l'huile d'arachide du régime, effectuée antérieurement (Semporé et Bézard, 1977 et 1982) et de la comparer aussi à la structure glycéridique d'un tissu adipeux de rats qui avaient été soumis à un régime à base de beurre de karité (Sawadogo et Bézard, 1982 a, b), ou à un régime pauvre en lipides (Bugaut et Bézard, 1973).

\section{Matériel et méthodes.}

Dix rats mâles de souche Wistar provenant de l'élevage de la Station de Recherches sur la Qualité des Aliments de I'Homme (INRA, Dijon), âgés de 4 semaines et pesant en moyenne $73 \mathrm{~g}$, sont répartis en cages individuelles permettant de récolter les fèces.

IIs reçoivent pendant 17 semaines un régime semi-synthétique équilibré (Rocquelin et Cluzan, 1968) contenant 15 p. 100 en poids (30 p. 100 de l'apport énergétique) d'huile d'arachide, provenant de l'Institut des Corps Gras (ITERG, Paris). La composition en acides gras de l'huile est rapportée dans le tableau 1 (première ligne). La nourriture est consommée ad libitum et la quantité consommée déterminée quotidiennement; le poids des rats est déterminé chaque semaine.

Ils sont soumis à une expérience de bilan pendant les 8 premiers jours de la mise au régime (Rocquelin et Leclerc, 1969 ; Sergiel et Gabucci, 1980). Les fèces sont collectées chaque jour, réunies et entreposées à $+4{ }^{\circ} \mathrm{C}$ pour chaque rat. Les animaux sont pesés au début et à la fin de l'expérience. 
Les lipides fécaux sont extraits par la méthode de Toullec, Flanzy et Rigaud (1968), qui permet de séparer une fraction soluble (FS) et une fraction insoluble (FI) dans le chloroforme-méthanol 2:1 (V/V). Les acides gras sont analysés par chromatographie gaz-liquide (CGL) sous forme d'esters butyliques (Clément et Bézard, 1961).

Après ces 4 mois, on met les rats à jeun $10 \mathrm{~h}$, on les anesthésie à l'éther et on prélève sur chacun un fragment de tissu adipeux périrénal de $1 \mathrm{~g}$ et on réunit les 10 échantillons. On applique ensuite à ce "pool " de tissus adipeux les méthodes d'extraction et d'analyses qui ont été décrites en détail par ailleurs (Semporé et Bézard, 1977 ; Bézard et al., 1981 ; Sawadogo et Bézard, 1982 a). Nous en rappellerons néanmoins les points essentiels : les lipides totaux sont extraits par la méthode de Folch, Lees et Sloane-Stanley (1957).

La fraction triglycéridique est séparée sur colonne d'acide silicique et les triacylglycérols (TG) sont fractionnés en classes selon leur degré d'insaturation, sur couches minces de silice imprégnée de nitrate d'argent (Barrett, Dallas et Padley, 1963) en deux migrations. Une première sur plaques de $0,5 \mathrm{~mm}$ d'épaisseur de silice (Kiesel G, Merck) contenant 10 p. 100 en poids de nitrate d'argent (Merck), en présence du milieu hexane-éther-méthanol $(158 / 40,2 / 1,8$, en volumes), sur $5 \mathrm{mg}$ de TG, a permis de séparer 7 bandes. Une deuxième migration sur le même type de plaque mais avec un milieu légèrement plus polaire, hexaneéther-méthanol $\left(158 / 39,8 / 2,2\right.$, en volumes), a permis de séparer la $4^{\mathrm{e}}$ bande à partir du front de migration en deux bandes correspondant aux classes 002 et 111 (voir tabl. 2), tandis que la dernière bande, complexe également, n'a pu être séparée en classes.

Les bandes délimitées sous lumière ultra-violette $(350 \mathrm{~nm})$ après pulvérisation d'une solution alcoolique de 0,2 p. 100 de dichlorofluorescéine, ont été grattées et les TG extraits de la silice en présence de chloroforme-méthanol $190 / 10$, V/V) selon Bézard et Bugaut (1972). Après addition d'un standard interne, I'heptadécanoïque (17:0), afin de déterminer la proportion des différentes fractions, les acides gras des TG correspondant à chaque bande ont été analysés par CGL sur colonne d'acier inoxydable $(1,20 \mathrm{~m} \times 1 / 8 \mathrm{in.})$ remplie de diéthylèneglycol succinate $(20$ p. 100 en poids) fixé sur Chromosorb W 80-100 mesh (Applied Science Laboratories: $A S L)$; les TG ont été aussi analysés, directement, par CGL sur colonne de verre $(60 \mathrm{~cm} \times 1 / 8 \mathrm{in.})$ remplie de Gas Chrom. Q 100-120 mesh (ASL) imprégné de 3 p. 100 de diméthylpolysiloxane (ASL). Les autres conditions des analyses par CGL ont été rapportées antérieurement (Semporé, et Bézard, 1977).

Les TG de chaque bande ont ensuite été soumis à une hydrolyse par la lipase pancréatique de Rat, afin de déterminer l'acide gras estérifié en position interne, selon Luddy et al. (1964), dans des conditions rapportées en détail par ailleurs (Bézard et al., 1981). On utilise un tampon Tris (trihydroxyméthylaminométhane) à $\mathrm{pH} 8$, contenant du calcium, en présence de $5 \mathrm{mg}$ de TG, émulsionnés par ultrasons en présence de taurocholate de sodium, et de $100 \mu \mathrm{l}$ de suc pancréatique de Rat, à $40^{\circ} \mathrm{C}$ pendant $1 \mathrm{~min}$, permettant une hydrolyse partielle de 45 p. 100 des TG présents. Après extraction, les lipides sont séparés sur cou- 
ches minces de silice (Kieselgel $G$, Merck) de 0,25 mm d'épaisseur, en présence du milieu hexane-éther-acide acétique-méthanol $(90 / 20 / 2 / 3$, en voiumesi.

Les acides gras des monoacylglycérols (MG), comportant plus de 97 p. 100 de 2-MG, et des diglycérides (DG), sont analysés par CGL.

Les DG sont par ailleurs acétylés en présence d'anhydride acétique et pyridine et fractionnés selon leur degré d'insaturation sur couches minces au nitrate d'argent. Les acides gras des différentes classes ainsi isolées sont analysés par CGL.

A partir des résultats obtenus lors de l'analyse des acides gras et des TG des différentes classes de TG, il est possible de déterminer mathématiquement la composition en types de TG du tissu adipeux analysé (Bézard, Bugaut et Clément, 1971 ; Semporé et Bézard, 1977 ; Sawadoge et Bézard, 1982 a). A partir, en plus, des résultats de l'analyse des acides gras des MG formés au cours de la lipolyse, en s'aidant, le plus souvent, de la composition en acides gras d'une ou deux classes des DG formés, il est possible de déterminer mathématiquement la composition en isomères de TG (Bézard et al., 1981 ; Semporé et Bézard, 1982 ; Sawadogo et Bézard, 1982 b). Rappelons qu'un type de TG comprend toutes les molécules qui présentent les mêmes trois acides gras constitutifs, alors que les isomères comportent, en plus, le même acide gras en position interne.

\section{Résultats.}

\section{Utilisation digestive des acides gras de l'huile d'arachide du régime.}

L'étude de la consommation de nourriture et de gain de poids des animaux tout au long des 4 mois de régime, a montré que celui-ci a été parfaitement accepté et a assuré une croissance normale, puisque le poids des animaux est passé de $73 \mathrm{~g}$ en moyenne à $562 \mathrm{~g}$, pour une consommation moyenne de $15 \mathrm{~g}$ de matière sèche par jour.

L'étude des bilans effectuée pendant 8 jours au moment de la mise au régime a montré (tabl. 1) que, pendant cette durée, la quantité d'acides gras ingérés a été de $10,7 \mathrm{~g}$ par rat, la quantité excrétée avec les fèces de $0,8 \mathrm{~g}$, soit un CUD apparent de 92,5 p. 100 , analogue à celui observé pour la matière sèche du régime, soit 92,2 p. 100. II apparaît toutefois que le CUD apparent n'est pas le même pour chacun des acides gras : il est très élevé pour les acides oléique (18:1) et linoléique (18:2), plus faible pour l'éicosénoïque (20:1); pour la série des acides gras saturés (18:0 à 24:0), le CUD est nettement moins élevé, particulièrement pour les acides à très longue chaîne (22:0 et 24:0). Ces acides gras saturés se retrouvent dans les fèces, en forte proportion dans la fraction dite insoluble, sous forme de savons.

\section{Composition en acides gras du tissu adipeux périrénal.}

L'analyse des acides gras du tissu adipeux périrénal des rats après 4 mois de régime (tabl. 2, dernière colonne), montre que l'on retrouve les 3 principaux aci- 


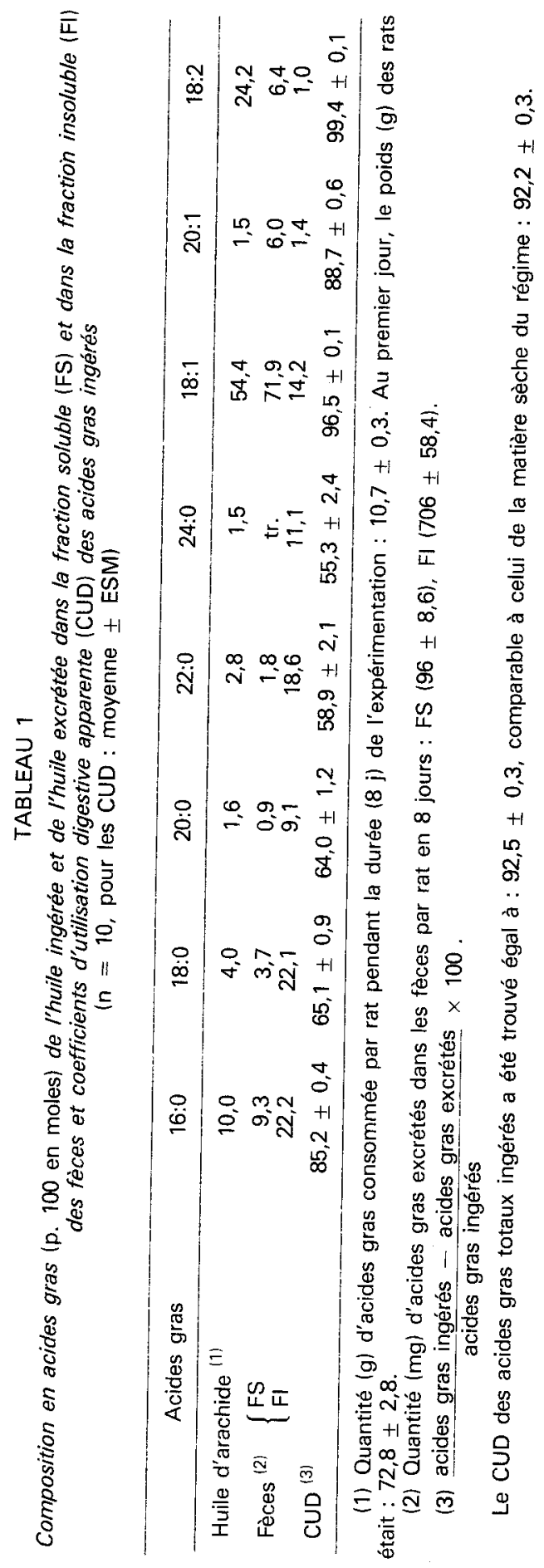




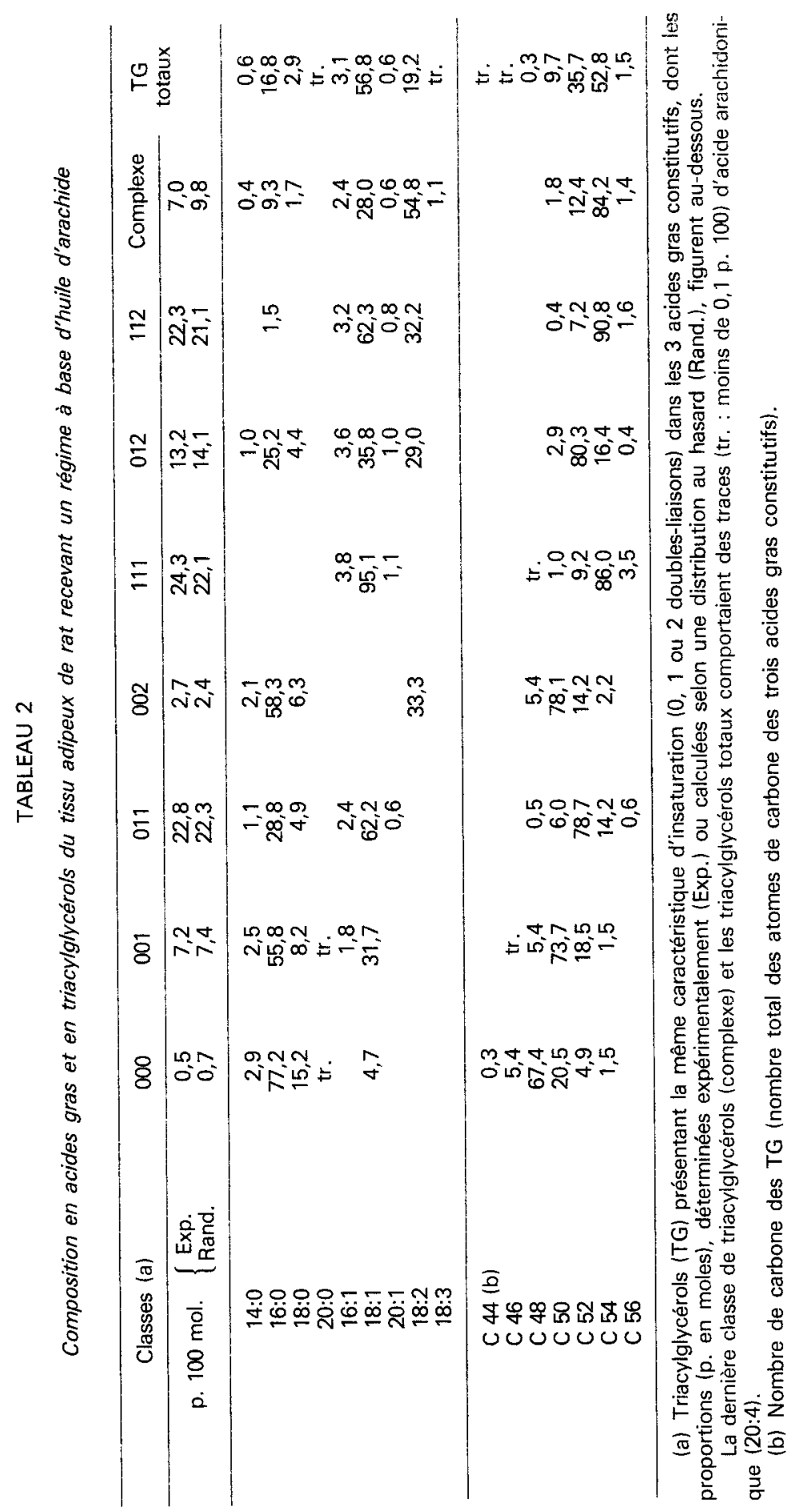


des gras de l'huie du régime dans ce tissu et dans des proportions aj soni relativement voisines. On note, en particulier, 56,8 p. $100 \mathrm{~d}$ 'acide óéiqu€, davantage que dans I'huile d'arachide apportée $(54,4$ p. 100). On notera aussi la forte proportion en acide linoléique (19,2 p. 100). II est remarquable enfin de voir que les acides gras à très longue chaîne (20:0 à 24:0), qui représentaient ensembie 6 p. 100 des acides gras totaux de l'huile d'arachide, ne se trouvent pas incorporés dans le tissu adipeux.

La richesse du tissu adipeux en acides gras en C 18 explique la richesse en TG en C 54, la présence d'acide palmitique en C 16 expliquant la présence de $T G$ en C 52.

Le fractionnement des TG selon l'insaturation montre (tabl. 2, lignes du haut) que les classes en proportions les plus importantes sont celles qui comportent de l'acide oléique. On notera en particulier la quantité élevée des TG trimonoinsaturés $(24,3$ p. 100), constituée essentiellement de trioléine puisque l'acide oléique représente 95,1 p. 100 des acides gras totaux de cette classe.

Les valeurs trouvées pour les pourcentages des 8 fractions isolées ne s'écartent que faiblement des valeurs calculées en supposant une répartition totalement au hasard sur les 3 positions du glycérol, d'un seul pool d'acides gras correspondant aux acides gras totaux (Litchfield, 1972).

\section{Acides gras en position interne.}

L'hydrolyse des TG par la lipase pancréatique dont l'action est spécifique des acides gras situés sur les positions externes du glycérol (Mattson et al., 1952), permet de connaître les acides gras qui sont estérifiés en position interne et qui sont ceux des 2-monoacylglycérols formés.

La lipolyse effectuée sur les TG totaux du tissu adipeux périrénal montre (tabl. 3, première ligne) que 91 p. 100 des acides gras en position interne sont des acides insaturés, alors que, dans les acides gras totaux des TG, ils ne représentaient que 80 p. 100 ; la différence n'est toutefois pas très importante. La localisation préférentielle peu marquée de l'acide oléique pour la position interne s'explique par la forte quantité de trioleoylglycérol qui comporte une molécule $d^{\prime}$ acide oléique en position interne pour deux en position externe.

Au niveau des différentes fractions, on retrouve aussi une localisation préférentielle des acides insaturés en position interne, de l'acide linoléique en particulier, comparée à celle des acides saturés. Ainsi, dans la classe 012, plus de la moitié des acides gras en position 2 est de l'acide linoléique et moins de un dixième des acides saturés. Cette localisation préférentielle des acides insaturés en position interne est particulièrement évidente lorsque ceux-ci sont en compétition uniquement avec des acides saturés, tels que dans les classes 001 et 002 , où les deux tiers environ des acides gras en position 2 sont représentés par le seul acide insaturé présent. Quand deux molécules d'acide insaturé sont en compétition avec une seule d'acide saturé (classe 011), cette proportion s'élève à près de neuf dixièmes. Comparé à l'acide oléique, l'acide linoléique est plus fréquemment trouvé en position interne, comme on peut le voir dans la classe 012. Quand il est en compétition avec deux molécules d'acide oléique (classe 112), les deux acides gras se partagent également la position interne. 


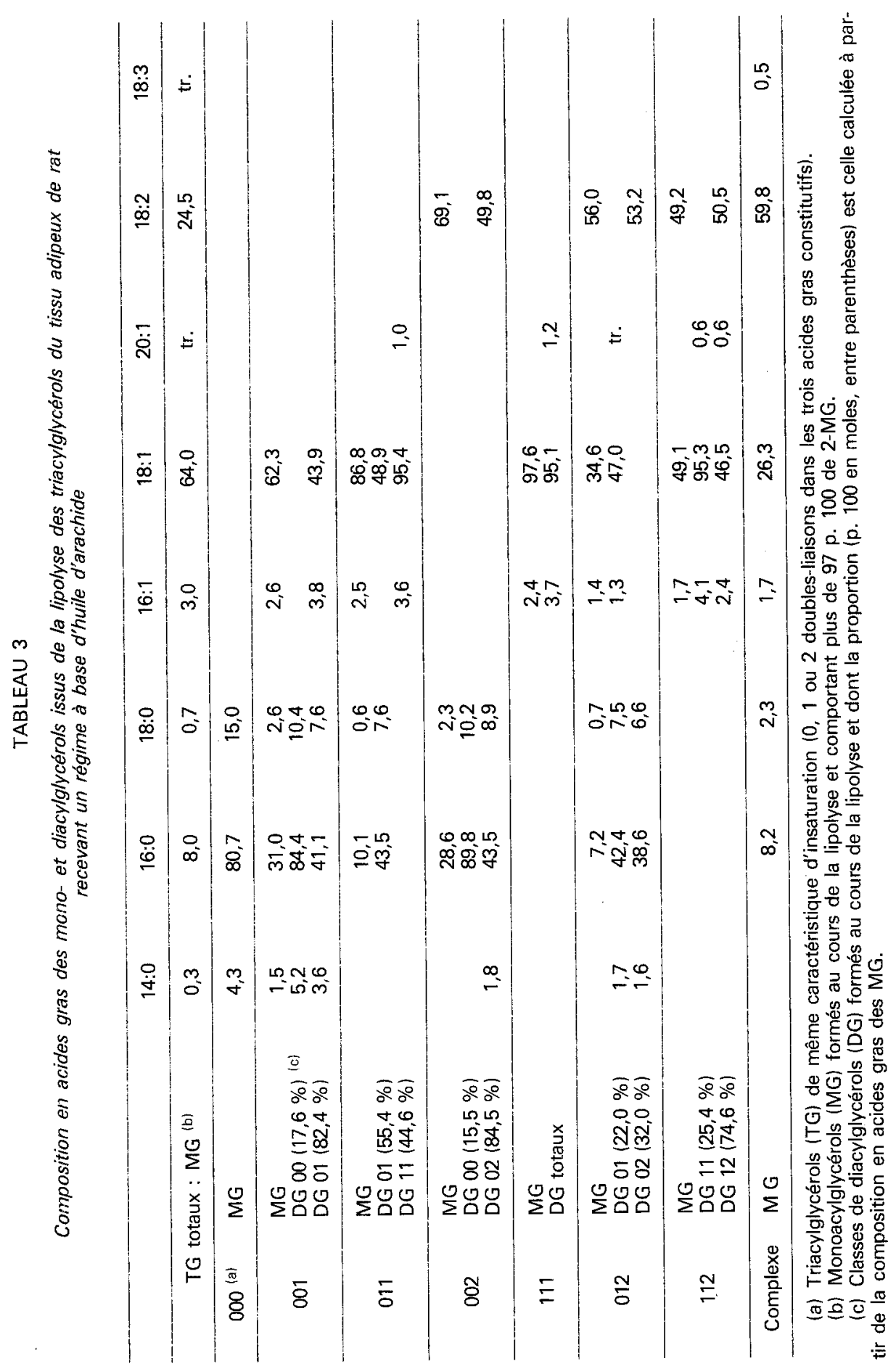


Pour cinq fractions, les diacylglycérols formés au cours de la lipolyse ont été séparés selon leur degré d'insaturation et les acides gras des fractions isolées ont été analysés, afin d'apporter des données expérimentales supplémentaires nécessaires au calcul de la composition en isomères (Bézard et al., 1981). Le pourcentage de ces fractions est celui calculé à partir de la composition des monoacylglycérols (Bézard et al., 1981 ; Semporé et Bézard, 1982).

\section{Composition en isomères de triacylglycérols.}

A partir de la composition en acides gras des MG formés au cours de la lipolyse de chaque classe de TG et dans quelques cas en plus, à partir d'une ou de deux classes de DG, il est possible de calculer une composition détailiée en isomères de TG (Bézard et al., 1981 ; Semporé et Bézard, 1982). Un isomère de TG comporte au maximum deux molécules de TG dont seuls les acides gras en positions 1 et 3 ont été permutés, l'acide gras en position interne étant le même.

Les résultats obtenus figurent dans le tableau 4 où les isomères ont été classés dans l'ordre décroissant des pourcentages. On peut voir qu'il existe 14 isomères seulement dont la proportion est supérieure à $1 \mathrm{p}$. 100 et ils représentent ensemble plus de 80 p. 100 des TG totaux du tissu adipeux. Le trioleoylglycérol, qui est le TG principal, compte pour plus du cinquième. Les quatre premiers isomères dont le pourcentage est, soit sensiblement égal, soit supérieur à 10, représentent à eux seuls plus de la moitié $(55,9$ p. 100) des TG totaux. Ils comprennent 9 molécules d'acide oléique sur les 12 molécules d'acides gras présentes. Dans ces 14 isomères, l'acide oléique occupe la position centrale 7 fois, en particulier dans les 3 premiers, et l'acide linoléique 5 fois. Pour deux d'entre eux, on trouve l'acide palmitique en position interne, mais le plus important ne représente que 2,1 p. 100.

Viennent ensuite 15 isomères dont le pourcentage est situé entre 0,5 et 1 et qui ne représentent plus que 10,6 p. 100 des TG du tissu adipeux. L'acide oléique $y$ est présent 7 fois encore en position interne, l'acide linoléique 4 fois.

On trouve ensuite 31 isomères dont le pourcentage est compris entre 0,5 et 0,1 . Ils représentent ensemble seulement 5,3 p. 100.

Enfin, 24 autres isomères ont pu être identifiés et leur proportion calculée. Ils n'interviennent que pour un pourcentage compris entre 0,1 et 0,01 et ne représentent ensemble que 1,1 p. 100 des TG totaux du tissu adipeux périrénal. Ils n'ont pas été rapportés dans le tableau 4.

En regard des valeurs de pourcentages déterminées mathématiquement à partir des données expérimentales, on a porté dans le tableau 4 celles calculées en supposant une distribution au hasard de deux pools d'acides gras, l'un pour la position interne, dont la composition est celle des 2-MG issus de la lipolyse des TG totaux, et l'autre pour les positions externes dont la composition se détermine par différence avec celle des TG de départ (Litchfield, 1972). On peut voir que les valeurs trouvées sont relativement voisines des valeurs expérimentales. En particulier, l'ordre d'importance quantitative est le même dans les deux cas. La différence est de 16 p. 100 pour le trioleoylglycérol, le TG majeur ; elle s'élève néanmoins à près de $40 \mathrm{p}$. 100 pour le $4^{\mathrm{e}}$ isomère par ordre d'importance. 


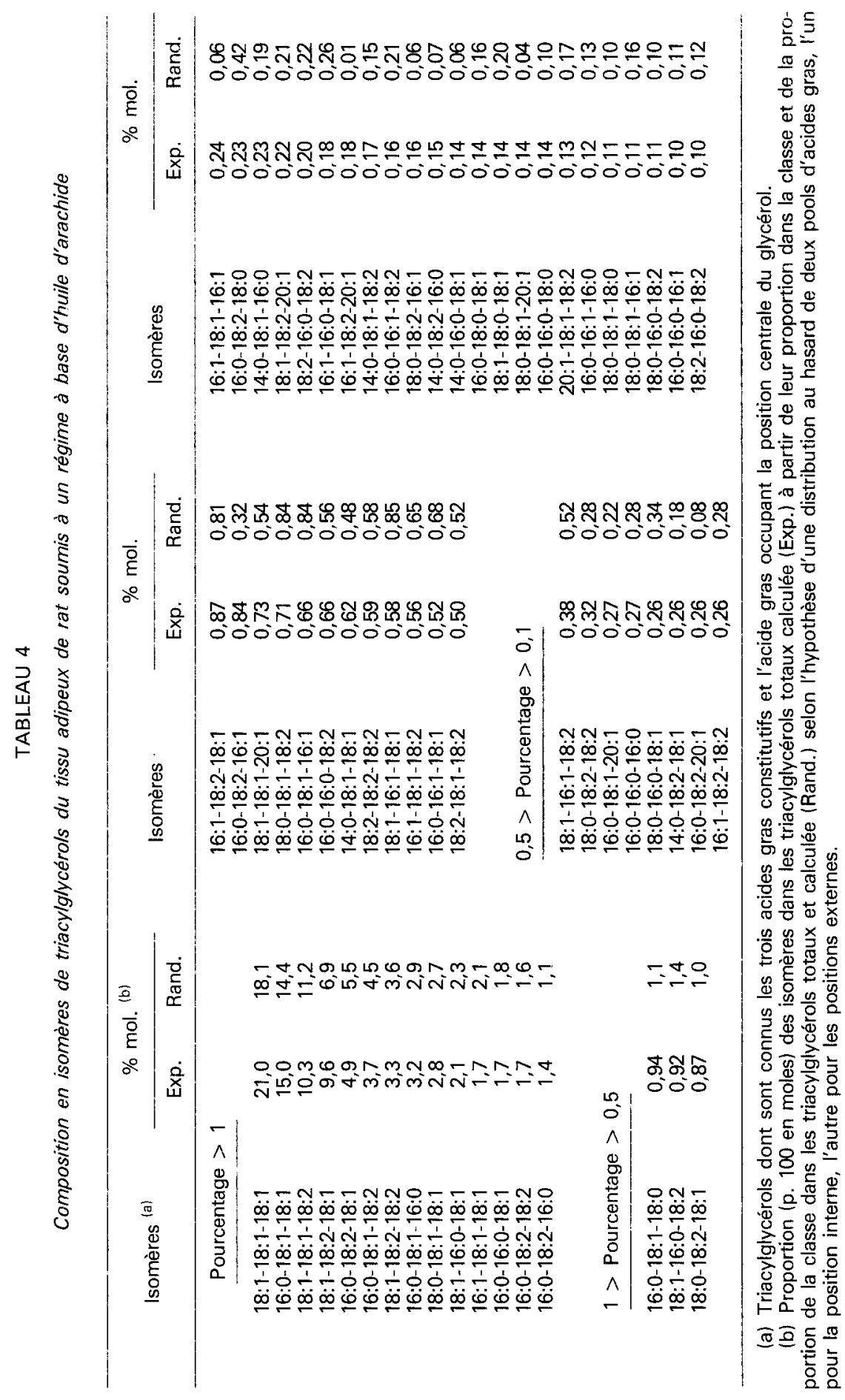




\section{Discussion.}

Si l'on compare tout d'abord la composition en acides gras du tissu adipeux périrénal de rat à celle de l'huile d'arachide du régime, la différence la plus évidente est l'absence d'acides gras saturés à très longue chaîne, en particulier des acides béhénique (22:0) et lignocérique (24:0), dans le tissu de réserve, alors que ces deux acides gras représentent 4,3 p. 100 de l'huile du régime ; I'acide arachidique (20:0) n'y est aussi présent qu'à l'état de traces (moins de 0,1 p. 100), alors qu'il représente 1,6 p. 100 des acides gras de l'huile.

L'étude de l'utilisation digestive des acides gras du régime montre que l'absorption intestinale de ces acides gras est très nettement inférieure à celle de l'acide oléique, par exemple, ou même de l'acide palmitique, confirmant des résultats déjà rapportés (Rocquelin et Leclerc, 1969). Cette particularité s'explique par la localisation exclusivement externe dans les molécules de TG de l'huile d'arachide des acides gras à très longue chaîne, qui sont donc libérés dans la lumière intestinale au cours de la digestion par la lipase pancréatique, formant en partie des savons insolubles qui ne sont pas absorbés; en revanche, les acides gras en position interne sont absorbés principalement sous forme de 2-MG (Clément, 1980). Néanmoins, plus de la moitié de ces acides gras disparaissent de la lumière intestinale, comme l'indiquent les CUD, et ils sont très vraisemblablement absorbés. Dans l'entèrocyte, ils seront estérifiés en position externe dans les nouvelles molécules de TG synthétisées à partir des 2-MG absorbés qui, eux, comportent presque exclusivement des acides insaturés (Semporé et Bézard, 1982).

Dans ces TG retrouvés dans les chylomycrons lymphatiques, les acides gras à très longue chaîne pourraient résister, au moins partiellement, à l'hydrolyse par la lipoprotéine lipase et la lipase hormono-sensible du tissu adipeux, comme cela a été montré pour l'acide phytanique, acide ramifié à 20 atomes de carbone (Ellingboe et Steinberg, 1972) ; ils pourraient aussi résister pareillement à d'autres lipases tissulaires, pouvant ainsi se déposer dans la paroi artérielle où leur pouvoir détergent serait susceptible de provoquer des lésions. Cette interprétation a aussi été avancée par d'autres auteurs (Manganaro et al., 1981).

L'interestérification de I'huile d'arachide, distribuant les acides gras constitutifs au hasard entre les 3 positions du glycérol, apporte $1 / 3$ des acides gras à très longue chaîne en position centraie, ceux-ci étant facilement absorbés sous forme de 2-MG. Mais, dans les TG resynthétisés dans l'entérocyte à partir de ces 2-MG, ces acides gras se trouvent localisés en position centrale, et ils résisteraient moins à l'hydrolyse par les lipases tissulaires, pouvant en particulier s'accumuler dans le tissu adipeux, ne persistant pas, de ce fait, dans la circulation générale. Ainsi pourrait s'expliquer que I'huile d'arachide « randomisée » voit son pouvoir athérogène fortement diminué chez les animaux (Kritchevsky et al., 1973). La raison de l'athérogénicité de l'huile d'arachide pourrait donc bien résider, non pas dans la teneur elle-même en acides gras saturés à très longue chaîne, mais plutôt dans leur localisation externe dans les molécules de TG, 
c'est-à-dire dans la structure de ces molécules, comme cela avait èté primitivement suggéré (Kritchevsky et al., 1971).

Pour expliquer l'absence d'acides gras à très longue chaîne dans le tissu adipeux, on ne peut toutefois exclure une transformation de ces acides gras dans l'organisme, le foie en particulier, par exemple un raccourcissement en acide palmitique. On ne peut non plus exclure une incorporation préférentielle des acides gras en C 22 et C 24 dans certains phospholipides d'organes, tels que les sphingomyélines. Dans ce. cas, le pouvoir athérogène de I'huile d'arachide devrait trouver une autre explication que celle suggérée plus haut et qui pourrait concerner d'autres constituants que les triglycérides et leurs acides gras.

Une situation analogue peut être observée dans le beurre de karité qui comporte 44 p. 100 d'acide stéarique (et 45 p. 100 d'acide oléique), mais situé presque exclusivement en position externe (Sawadogo et Bézard, 1982 a). L'analyse du tissu adipeux périrénal de rats soumis pendant 4 mois à un régime comportant 15 p. 100 en poids de beurre de karité, montre que l'incorporation de l'acide stéarique dans les tissus de réserve est de faible importance, bien que les $3 / 4$ soient absorbés dans l'intestin (Sawadogo et Bézard, 1982 b). Il serait intéressant de savoir si cette graisse, qui est consommée en particulier par les populations africaines, présente aussi un pouvoir athérogène chez les animaux. Nous n'avons pas connaissance qu'une telle étude ait été faite.

En dehors de cette différence entre le tissu adipeux de rat et l'huile d'arachide du régime concernant les acides gras à très longue chaîne, il existe en revanche de grandes ressemblances entre les TG de l'un et de l'autre. On retrouve, tout d'abord, les mêmes trois acides gras principaux (18:1, 18:2 et 16:0) de I'huile dans le tissu de réserve et dans des proportions qui ne sont pas très différentes ; en particulier, la proportion d'acide oléique est sensiblement la même, de l'ordre de 55 p. 100 (Semporé, et Bézard, 1977).

La localisation de ces acides gras dans les molécules de TG présente aussi des similitudes assez grandes. Toutefois, dans le tissu adipeux, l'acide linoléique est moins préférentiellement localisé en position interne que dans I'huile d'arachide, tandis que l'acide palmitique se retrouve dans cette position dans le tissu adipeux en proportion non négligeable, beaucoup plus élevée que dans l'huile d'arachide (Semporé et Bézard, 1982).

Concernant la composition en isomères de TG, on trouve, là aussi, de grandes analogies. L'on considérera seulement les isomères en quantité supérieure à 1 p. 100, soit 17 dans I'huile d'arachide, représentant 83 p. 100 des TG totaux (Semporé et Bézard, 1982) et 14 dans le tissu adipeux, représentant 82 p. 100. On trouve, parmi ceux-ci, 10 isomères qui sont les mêmes et qui représentent respectivement 72 p. 100 de l'huile d'arachide et 75 p. $100 \mathrm{du}$ tissu adipeux. En particulier, on trouve une forte proportion de trioleoylglycérol dans les deux cas, 25 et 21 p. 100 respectivement. II existe, pour les suivants, des inversions dans i'importance des différents isomères qui traduisent les différences de localisation en position interne des acides oléique et linoléique signalées plus haut. Les isomères de l'huile d'arachide, comportant un acide gras à très longue chaîne, ne sont pas retrouvés dans le tissu adipeux, comme il fallait s'y attendre.

Cette similitude marquée dans la structure des TG des deux origines ne 
signifie pas évidemment que la structure des TG de l'huile ingérée est intégralement conservée au cours des processus de digestion, d'absorption intestinales et de mise en réserve dans l'adipocyte. Elle l'est, au moins en partie, et, concernant l'acide gras en position centrale, après absorption, dans les TG lymphatiques (Clément, Bézard et Courel, 1965); elle peut se conserver après l'action de la lipoprotéine lipase du tissu adipeux, qui est spécifique de la position 1 (Morley et Kuksis, 1972). La resynthèse de TG dans l'adipocyte utilise les acides gras libérés par la lipoprotéine lipase et qui étaient ceux utilisés par l'entérocyte pour resynthétiser les TG, c'est-à-dire ceux provenant principalement de l'huile ingérée (Clément, 1980). On peut s'expliquer ainsi qu'on puisse retrouver en grande partie dans le tissu adipeux les mêmes isomères que dans l'huile du régime, d'autant plus que les processus de mise en réserve des TG ingérés ne doivent pas être masqués par des remaniements de structure qui pourraient se produire si le régime était interrompu. On retrouve donc là une propriété déjà bien connue du tissu adipeux, dont la composition se rapproche de celle des graisses du régime (Hirsch, 1962 ; Bézard et Bugaut, 1969), mais elle est démontrée, dans ce travail, à un niveau beaucoup plus bas d'organisation moléculaire des acides gras dans les triacylglycérols.

Cette propriété n'est toutefois pas générale et dépend du type de graisse ingérée par l'animal. Ainsi, avec le beurre de karité (Sawadogo et Bézard, 1982 a et b), l'isomère principal de la graisse (42 p. 100 des TG totaux), qui contient deux molécules d'acide stéarique en positions externes et une d'acide oléique en position interne, n'est pratiquement pas retrouvé $(0,4 \mathrm{p} .100$ seulement) dans le tissu adipeux. En revanche, on y trouve 28 p. 100 de trioleoylglycérol, représentant seulement 6 p. 100 dans le beurre de karité, ce qui peut suggérer que l'acide stéarique, absorbé en proportion non négligeable, a été transformé en acide oléique, soit directement par désaturation, soit après dégradation complète, et synthèse de novo.

En revanche, malgré des compositions en acides gras très différentes des deux corps gras, huile d'arachide et beurre de karité, les tissus adipeux correspondants montrent des compositions en TG très comparables. En effet, les deux TG majeurs sont les mêmes et représentent respectivement 36 p. 100 et 50 p. 100 des TG totaux des tissus adipeux; en outre, parmi les 14 et 16 TG les plus importants respectivement, 11 sont les mêmes et représentent 76 p. 100 dans le tissu adipeux de rat nourri à l'huile d'arachide et 80 p. 100 dans celui de rat nourri au beurre de karité.

Lorsque le régime apporte peu d'acides gras (Bézard et Bugaut, 1972 ; Bugaut et Bézard, 1973), le tissu adipeux et le foie en synthétisent, principalement de l'acide palmitique et de l'acide oléique et, dans ce cas (Bugaut et Bézard, 1973), on trouve une forte proportion de TG associant ces 2 acides gras, le type de TG majeur étant le 16:0 18:2 18:1 (près de 17 p. 100) ; mais, en revanche, il y a très peu $(2,7$ p. 100$)$ de trioleoylglycérol.

Ces divers résultats pourraient signifier qu'il existe une régulation dans la synthèse des TG par le tissu adipeux de Rat, vraisemblablement au niveau des acyltransférases. Toutefois, lorsque l'huile du régime comporte une proportion importante d'un acide gras facilement incorporé dans les TG du tissu adipeux, tel 
que l'acide oléique dans l'huile d'arachide ou le beurre de karité, les chances de faire apparaître des identités de structure entre le corps gras alimentaire et les graisses de réserve augmentent statistiquement, par conséquent, sans intervention régulatrice. Si, au contraire, l'acide gras du régime est l'objet d'une transformation métabolique intense, on ne retrouve plus dans les TG de réserve la structure observée dans les TG ingérés. C'est le cas de l'acide laurique (12:0), présent principalement en position centrale dans l'huile de coprah et retrouvé en position externe dans les TG du tissu adipeux (Clément, Bézard et Courel, 1965).

Recu en mai 1982. Accepté en août 1982.

Remerciements. - Nous remercions J. Causeret et ses collaborateurs, Station de Recherches sur la Qualité des Aliments de I'Homme, INRA, Dijon, qui nous ont apporté le matériel, les animaux et les techniques pour l'étude des bilans digestifs. Nous remercions aussi I'Institut des Corps Gras (ITERG), Paris, qui nous a fourni l'huile d'arachide.

\section{Références}

BARRETT C. B., DALLAS M. S. J., PADLEY F. B., 1963. The quantitative analysis of triglyceride mixtures by thin layer chromatography on silica impregnated with silver nitrate. J. am. Oil Chem. Soc., 40, 580-584.

BEZARD J., BUGAUT M., 1969. Gas-liquid chromatography of fatty acids and triglycerides from rat adipose tissue. J. Chromatog. Sci., 7, 639-644.

BÉZARD J., BUGAUT M., CLÉMENT G., 1971. Triglyceride composition of coconut oil. J. am. Oil Chem. Soc., 48, 134-139.

BEZZARD J., BUGAUT M., 1972. The component triglycerides of rat adipose tissue. I. As studied after fractionation into classes by silver ion - thin layer chromatography. J. Chromatog. Sci., 10, 451-462.

BÉZARD J., SEMPORÉ G., DESCARGUES G., SAWADOGO A., 1981. Further studies on the determination of the 2-isomers of triglycerides from natural fats. Fette Seifen Anstrich., 83. 17-23.

BRISSON G., 1982. Graisses insaturèes contre graisses saturées, 107-125. In BRISSON G., Lipides et nutrition humaine. Les Presses de I'Université Laval, Québec, et Masson, Paris.

BUGAUT M., BÉZARD J., 1973. The component triglycerides of rat adipose tissue. II. As studied after fractionation of classes into groups by gas-liquid chromatography. $J$. Chromatog. Sci., 11, 36-46.

CLÉMENT '., 1980. Intestinal absorption of triacylglycerols. Reprod. Nutr. Dévelop., 20 , 1285-1307.

CLEMENT G., BEZARD J., 1961. Technique de dosage par chromatographie gaz-liquide, du butanoïque au docosanoïque. C.R. Acad. Sci., Paris, sér. D, 253, 564-566.

CLEMENT J., BEZARD J., COUREL E., 1965. Remaniements effectués au niveau digestif et au niveau du tissu adipeux de la structure des triglycérides après administration au Rat d'un régime à base d'huile de coprah. Biochim. biophys. Acta, 106, 25-33.

ELLINGBOE J., STEINBERG D., 1972. Differential susceptibility of phytanyl and palmityl ester bonds to enzymatic hydrolysis. Biochim. biophys. Acta, 270, 92-102.

FOLCH J., LEES M., SLOANE-STANLEY G. H., 1957, A simple method for the isolation and purification of total lipids from animal tissues. J. biol. Chem., 226, 497-509. 
FOOD AGRICULTURAL ORGANIZATION, 1977. Le rôle des graisses et huiles alimentaires en nutrition humaine, 1-99. Rapport d'un groupe d'experts, Rome.

GRESHAM G. A., HOWARD A. N., 1960. The independent production of atherosclerosis and thrombosis in the rat. Brit. J. exp. Pathol., 41, 395-402.

HIRSCH J., 1962. Composition of adipose tissue, 79-125. In KINSELL L. W., Adipose tissue as an organ. Charles C. Thomas, Publ., Springfield, Illinois, USA.

HOKES J. C., WORTHINGTON R. E., 1979. Structure of peanut oil triacylglycerols from cultivars of diverse genetic background. J. am. Oil Chem. Soc., 56, 953-956.

HORNSTRA G., 1975. Specific effects of types of dietary fat on arterial thrombosis, 303-330. In VERGROESEN A. J., The role of fats in human nutrition. Acad. Press, London, New York, San Francisco.

IMAI H., LEE K. T., PASTORI S., PONLILIO E., FLORENTIN R., THOMAS W. A., 1966. Atherosclerosis in rabbits. Architectural and subcellular alterations of smooth muscle cells of aortas in response to hyperlipemia. Exp. mol. Pathol., 5, 273-310.

KRITCHEVSKY D., TEPPER S. A., VESSELINOVITCH D., WISSLER R. W., 1971. Cholesterol vehicle in experimental atherosclerosis. 11. Peanut oil. Atherosclerosis, 14, 53-64.

KRITCHEVSKY D., TEPPER S. A., VESSELINOVITCH D., WISSLER R. W., 1973. Cholesterol vehicle in experimental atherosclerosis. 13. Randomized peanut oil. Atherosclerosis, 17, 225243.

KRITCHEVSKY D., TEPPER S. A., KIM H. K., STORY J. A., VESSELINOVITCH D., WISSLER R. W., 1976. Experimental atherosclerosis in rabbits fed cholesterol-free diets. 5. Comparison of peanut, corn, butter, and coconut oils. Exp. mol. Pathol., 24, 375-391.

LITCHFIELD C., 1972. Distribution of fatty acids in natural triglyceride mixtures, 233-264. In LITCHFIELD C., Analysis of trig/ycerides. Acad. Press, New York and London.

LUDDY F. E., BARFORD R. A., HERB S. F., MAGIDMAN P., RIEMENSCHNEIDER R. W., 1964. Pancreatic lipase hydrolysis of triglycerides by a semimicro technique. J. am. Oil Chem. Soc., 41, 693-696.

MANGANARO F., MYHER J. J., KUKSIS A., KRITCHEVSKY D., 1981. Acylglycerol structure of genetic varieties of peanut oils of varying atherogenic potential. Lipids, 16, 508-517.

MATTSON F. H., BENEDICT J. H., MARTIN J. B., BECK L. W., 1952. Intermediates formed during the digestion of triglycerides. J. biol. Chem., 219, 735-740.

MORLEY N., KUKSIS A., 1972. Positional specificity of lipoprotein lipase. J. biol. Chem., 247. 6389-6393.

MYHER J. J., MARAI L., KUKSIS A., KRITCHEVSKY D., 1977. Acylglycerol structure of peanut oils of different atherogenic potential. Lipids, 12, 775-785.

ROCQUELIN G., CLUZAN R., 1968. L'huile de colza riche en acide érucique et l'huile de colza sans acide érucique : valeur nutritionnelle et effets physiologiques chez le Rat. Ann. Biol. anim. Bioch. Biophys., 8, 395-406.

ROCQUELIN G., LECLERC J., 1969. L'huile de colza riche en acide érucique et l'huile de colza sans acide érucique. II. Utilisation digestive comparée chez le Rat. Ann. Biol. anim. Bioch. Biophys., 9, 413-426.

SANDERS T. H., 1979. Varietal differences in peanut triacylglycerol structure. Lipids, 14, 630-633.

SAWADOGO K., BÉZARD J., 1982 a. Etude de la structure glycéridique du beurre de karité. Oléagineux, 37, 69-74.

SAWADOGO K. A., BÉZARD J. A., 1982 b. Structure des triglycérides du tissu adipeux de rat soumis à un régime à base de beurre de karité. Oléagineux, 37, 247-253.

SCOTT R. F., MORRISON E. W., THOMAS W. A., JONES R., NAM S. C., 1964. Short-term feeding of unsaturated versus saturated fat in the production of atherosclerosis in the rat. Exp. mol. Pathol., 3, 421-443.

SCOTT R. F., MORRISON E. S., JARMOLYCH J., NAM S. C., KROMS M., COULSTON F., 1967. Experimental atherosclerosis in rhesus monkeys. Exp. mol. Pathol., 7, 34-57.

SEMPORÉ G., BÉZARD J., 1977. Les triglycérides de I'huile d'arachide. Rev. fr. Corps Gras, 24, 611-621.

SEMPORÉ G., BÉZARD J., 1982. Triacylglycerol structure of an african peanut oil. J. am. Oil Chem. Soc., 59, 124-129. 
SERGIEL J. P., GABUCCI L., 1980. Utilisation Digestive Comparée et formes d'excrétion fécale chez le Rat, des acides érucique et brassidique estérifiés dans différentes structures glycéridiques. Reprod. Nutr. Dévelop., 20, 1415-1427.

TOULLEC R., FLANZY J., RIGAUD J., 1968. Dosage des lipides des fèces. Extraction séparée, importance et composition en acides gras des lipides non saponifiés et de ceux des complexes insolubles. Ann. Biol. anim. Bioch. Biophys., 8, 281-289.

VAN PEE W., VAN HEE J., BONI L., HENDRIKX A., 1979. Positional distribution of the fatty acids and triglycerides of the oil of some African peanut varieties. J. Am. Oil Chem. Soc., 56, 901-903.

VERGROESEN A. J., GOTTENBOS J. J., 1975. The role of fats in human nutrition: an introduction, 1-32. In VERGROESEN A. J. The role of fats in human nutrition. Acad. Press, London, New York, San Francisco.

VESSELINOVITCH D., GETZ G. S., HUGHES R. H., WISSLER R. W., 1974. Atherosclerosis in the rhesus monkey fed three food fats. Atherosclerosis, 20, 303-321. 